\title{
OH/IR STARS IN THE LARGE MAGELLANIC CLOUD: THE OBSERVATIONS
}

\author{
P.R. WOOD, M.S. BESSELL, S.M.G. HUGHES, A.R. HYLAND \\ Mt. Stromlo \& Siding Spring observatories, ANU \\ Woden, ACT, 2600 \\ Australia
}

\author{
J.B. WHITEOAK, F.F. GARDNER, R.E. OTRUPCEK \\ Australia Telescope National Facility $\dagger$ \\ Epping, NSW, 2121 \\ Australia
}

$\dagger$ The Australia Telescope National Facility is operated in association with the Division of Radiophysics by CSIRO

In 1985 we began a search for OH/IR objects in the Magellanic Clouds. The first detection was reported by Wood, Bessell \& Whiteoak (1986). Subsequent searches have yielded several of these objects and other highly-evolved stars obscured by thick circumstellar shells.

The 1612-MHz OH observations were made using the Parkes 64-m radio telescope. Most of the observations utilized a dual-channel cryogenic receiver providing a system temperature of around $38 \mathrm{~K}$ on cold sky. The $\mathrm{OH}$ spectra were obtained with the Parkes digital correlator split into 512-channel segments. Bandwidths of $2 \mathrm{MHz}$ provided a resolution of $7.8 \mathrm{kHz}$ (equivalent to $1.5 \mathrm{~km} \mathrm{~s}^{-1}$ in radial velocity) after Hanning smoothing. The mode of observation has been described by Whiteoak and Gardner (1976). Typically, an integration period of 60 minutes was used; this yielded a detection limit $(3 \sigma)$ of around $50 \mathrm{mJy}$ for an $\mathrm{OH}$ feature. Detected emission was reobserved with a $1-\mathrm{MHz}$ bandwidth. A search was also made for $1665-\mathrm{MHz} \mathrm{OH}$ emission.

Our basic sample of objects was selected from the IRAS point source catalog. A few Harvard variables known to be bright asymptotic giant branch (AGB) stars were also included. Objects with flux densities above $0.7 \mathrm{Jy}$ at $25 \mu \mathrm{m}$ wavelength $\left(\mathrm{S}_{25}\right)$ were chosen, provided that they satisfied $0.5>\log \left(\mathrm{S}_{25} / \mathrm{S}_{12}\right)>-0.1$ and were not obvious HII regions or clusters.

$1612 \mathrm{MHz}$ observations were obtained for 39 positions. Maser emission was detected in six cases, all in the LMC. Most of the profiles show a pair of narrow features typical of Galactic $\mathrm{OH} / \mathrm{IR}$ objects. Fig. 1 shows the profile containing the most intense emission. Emission at $1665 \mathrm{MHz}$ was also detected in two cases. Table 1 lists the detected $\mathrm{OH} / \mathrm{IR}$ objects and associated $\mathrm{OH}$ results. At $1612 \mathrm{MHz}$, the peak flux densities of the two main components are listed. $\mathrm{V}_{\text {exp }}$, the stellar wind expansion velocity, is assumed to be half the velocity separation of the outer edges of these components, and $\mathrm{V}^{*}$ is the mean value of the corresponding velocities.

IRAS $05280-6910$ has atypical OH/IR spectra showing a series of narrow $1612-\mathrm{MHz}$ features between 248 and $295 \mathrm{~km} \mathrm{~s}^{-1}$ (Fig. 2) and a brighter $1665-\mathrm{MHz}$ feature at $240 \mathrm{~km} \mathrm{~s}^{-1}$. The spectra are not unlike those for the Galactic object IRAS 15405-4945 which te Lintel Hekkert et al. (1988) have suggested belongs to a small group of OH/IR stars showing bipolar mass loss. Pointing observations using the $1665 \mathrm{MHz}$ feature yielded a position of RA(1950) $=05 \mathrm{~h} 27 \mathrm{~m} 56 \mathrm{~s}$ $\pm 6 \mathrm{~s}, \operatorname{Dec}(1950)=-69^{\circ} 09^{\prime} 45^{\prime \prime} \pm 30^{\prime \prime}$. It is similar to the IRAS position for the object $(05 \mathrm{~h} 28 \mathrm{~m} 00 \mathrm{~s}$, $\left.-69^{\circ} 09^{\prime} 25^{\prime \prime}\right)$, and places the object near the centre of cluster NGC 1984.

IRAS sources were also observed with the 2.3-m telescope at Siding Spring Observatory and the Anglo-Australian Telescope (AAT). The limiting K magnitude was 11 for the 2.3 telescope, 12.5 for the AAT. An area within 30 arcsec of the selected IRAS position was searched. J, H and L' magnitudes were obtained for detected objects. Table 2 lists the detections. The intensities, in particular those of the detected OH/IR stars, were found to vary; Fig. 3 shows an example. All the periods are very long, exceeding 1000 days; this also occurs for some of the Galactic OH/IR stars (Herman \& Habing 1985; Engels et al. 1983). Assuming an LMC distance modulus of 18.5 , bolometric luminosities $\left(\mathrm{M}_{\mathrm{bol}}\right)$ were estimated from mean magnitudes.

Fig. 4 shows $\mathrm{M}_{\text {bol }}$ plotted against $\log$ period (P) for optically detected long-period variables in the LMC (Wood, Bessell \& Fox 1983; Reid, Glass \& Catchpole 1988; Hughes \& Wood 1990) and for the OH/IR objects of Table 3. It appears that the IRAS sources form longer-period extensions of both the supergiant and AGB sequences. 
The LMC objects appear to have expansion velocities about half those of their Galactic counterparts with similar luminosities. The terminal velocity of the stellar winds in late-type stars may be due to the effects of radiation pressure acting on grains in the circumstellar material, with the grains dragging the gas along with them (e.g. Knapp 1986; Zuckerman \& Dyck 1986; Jura 1986). This leads to an expansion velocity proportional to $\mathrm{k}^{1 / 2}$, where $\mathrm{k}$ is the opacity of the dusty material. Thus the smaller velocities in the LMC might reflect a lower abundance of grains.

\section{REFERENCES}

Engels,D., Kreysa,E., Schulz, G.V. \& Sherwood, W.A. 1983. Astr. Astrophys., 124,123.

Herman J. \& Habing, H.J. 1985. Astr. Astrophys. Suppl., 59, 523.

Hughes, S.M.G. \& Wood, P.R. 1990. Astron. J., 99, 784.

Jura, M. 1986. Irish Astron. J., 17, 322.

Knapp, G.R. 1986. Mitt. Astron. Ges., No. 67, 111.

Reid, N., Glass, I.S. \& Catchpole, R.M. 1988. Mon. Not. R. Astr. Soc., 232, 53.

te Lintel Hekkert, P., Habing, H.J., Caswell, J.L., Norris, R.P. \& Haynes, R.F. 1988. Astr.

Astrophys., 202, L19.

Whiteoak, J.B. \& Gardner, F.F. 1976. Mon. Not. R. Asdtr. Soc., 176, 25 P.

Wood, P.R., Bessell, M.S. \& Fox, M.W. 1983. Astrophys. J., 272, 99.

Wood, P.R., Bessell, M.S. \& Whiteoak, J.B. 1986. Astrophys. J. (Letters), 306, L81.

Zuckerman, B. \& Dyck, H.M. 1986. Astrophys. J., 304, 394.

Table 1. Detected LMC OH/IR Objects

\begin{tabular}{|c|c|c|c|c|}
\hline IRAS & $\begin{array}{c}S_{1612} \\
(\mathrm{Jy})\end{array}$ & $\begin{array}{c}S_{1665} \\
\text { (Jy) }\end{array}$ & $\begin{array}{l}V_{\exp } \\
\quad(\mathrm{km} \mathrm{s}\end{array}$ & $\mathrm{V}^{*}$ \\
\hline 00 & $0.14,0.03$ & $<0.04$ & 9.5 & 266 \\
\hline & $0.39,0.20$ & $0.08,0.13$ & 9.5 & 260 \\
\hline & $0.09,0.07$ & 0.31 & 22.5 & 272 \\
\hline & $0.24,0.13$ & $<0.06$ & 12.5 & 282 \\
\hline & $0.07,0.13$ & $<0.05$ & 12.0 & 312 \\
\hline 956 & $0.07,0.07$ & & 12.5 & 272 \\
\hline
\end{tabular}

Table 2. Near-IR Detections

IRAS

00477-7343 00521-7054 01039-7305

$04509-6922 \quad 04516-6902 \quad 04530-6916$

$04545-7000 \quad 04553-6825 \quad 04571-6954$

05216-6753 05244-6832 $05247-6941$

05261-6614 05294-7104 05298-6957

$05325-6743 \quad 05329-6708 \quad 05389-6922$
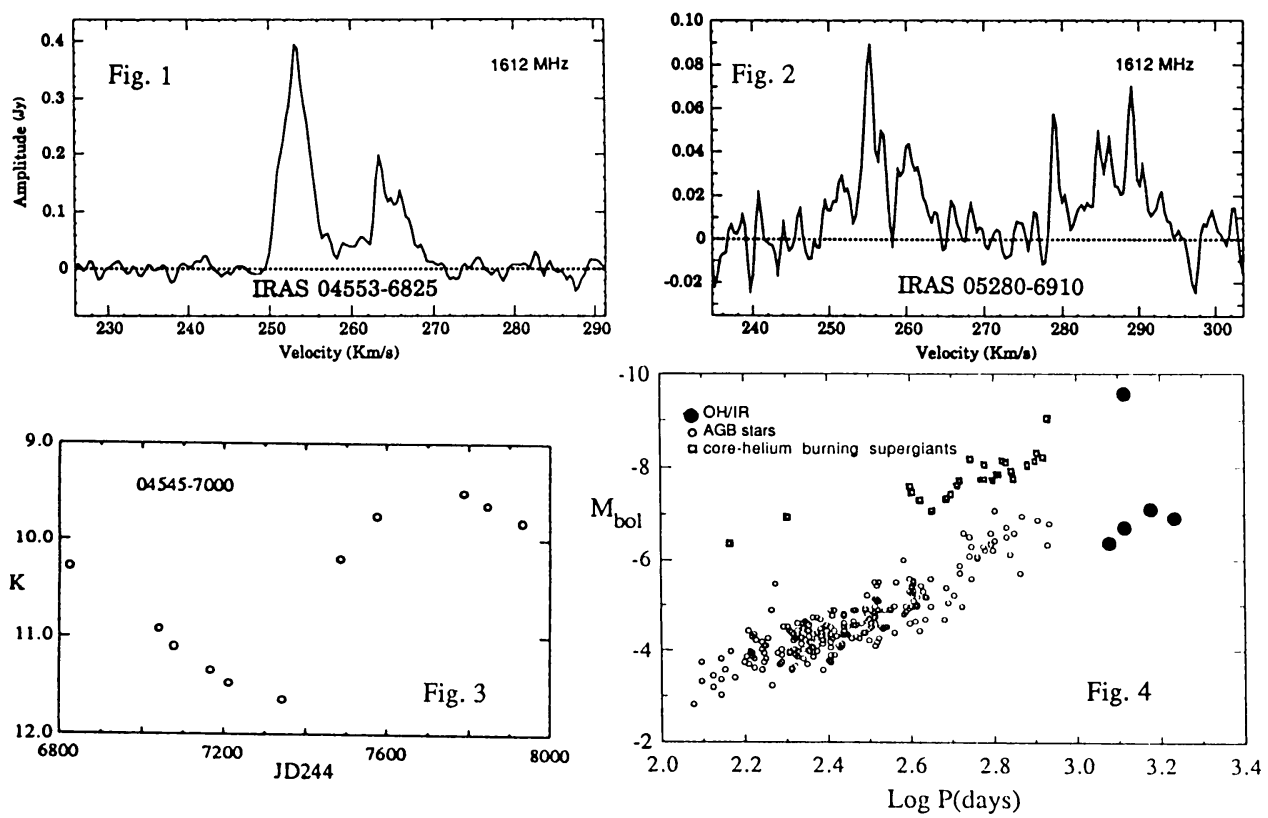